\title{
COMMUTANTS THAT DO NOT DILATE
}

\author{
DOUGLAS N. CLARK ${ }^{1}$
}

\begin{abstract}
The Lifting Theorem deals with dilation of the commutant of an operator $T_{1}$ on Hilbert space. In this note, counterexamples are given to generalizations of the theorem involving $N$ commuting operators $T_{1}, T_{2}, \cdots, T_{N}$.
\end{abstract}

In general terms, the Lifting Theorem for restricted shifts states that if $T$ is an operator commuting with the projection of the shift $S_{1}$ (on $H^{2}$ ) to one of its star-invariant subspaces, then $T$ may be dilated, without changing its norm, to an operator commuting with $S_{1}$. The theorem was first proved by Sarason [3], and has been extended by Sz.-Nagy and Foias to vector-valued $H^{2}$ spaces [4], and other, more general, situations [5].

To state the theorem more precisely, let us introduce some notation which at once suggests a different sort of generalization. Let $U^{N}$ denote the polydisk in $N$ complex variables $z_{1}, \cdots, z_{N}$. Let $\mathscr{H}$ be a separable Hilbert space with inner product $\langle\cdot, \cdot \cdot\rangle$, and let $H_{\mathscr{H}}^{2}\left(U^{N}\right)$ denote the $H^{2}$ space of $U^{N}$ based on $\mathscr{H}$. Thus an element of $H_{\mathscr{H}}^{2}\left(U^{N}\right)$ has the form

$$
f\left(z_{1}, \cdots, z_{N}\right)=\sum a_{J^{\prime}} z_{1}^{j_{1}} z_{2}^{j} \cdots z_{N}^{j_{N}}
$$

where the sum is over $J=\left(j_{1}, \cdots, j_{N}\right) \in Z_{+}^{N}$, where $a_{J} \in \mathscr{H}$, and where $\sum\left\|a_{J}\right\|^{2}<\infty$. Let $S_{1}, \cdots, S_{N}$ denote the shifts $\left(S_{j} f=z_{j} f\right)$ on $H_{\mathscr{H}}^{2}\left(U^{N}\right)$, let $M$ denote a subspace of $H_{\mathscr{H}}^{2}\left(U^{N}\right)$, invariant under $S_{1}, \cdots, S_{N^{\prime}}$, and define

$$
T_{j} f=P_{M^{\perp}} z_{j} f, \quad f \in M^{\perp}=H_{\mathscr{H}}^{2}\left(U^{N}\right) \ominus M .
$$

The above Lifting Theorem now states that, if $N=1$ and if $T$ commutes with $T_{1}$, then there is a dilation $S$ of $T$ which commutes with $S_{1}$ and which satisfies $\|S\|=\|T\|$.

The purpose of this note is to give examples of invariant subspaces $M$ in $H_{\mathscr{H}}^{2}\left(U^{2}\right)$ and $H^{2}\left(U^{3}\right)\left(=H_{C}^{2}\left(U^{3}\right), C\right.$ the complex numbers $)$ and of bounded operators $T$ on $M^{\perp}$, commuting with the $T_{j}$, but having no (bounded) dilation commuting with the $S_{j}$. Such a $T$ always has an

Presented to the Society, February 15, 1971; received by the editors March 1, 1971 and, in revised form, February 5, 1972.

AMS 1970 subject classifications. Primary 47A20; Secondary 47A45, 32A99, 30A80.

${ }^{1}$ Partially supported by NSF grant GP-11475.

(c) American Mathematical Society 1972 
unbounded dilation commuting with the $S_{j}$, as I proved in [1]. For an example involving a different dilation problem for commuting operators, see Parrott [2].

Our first example has to do with norms of dilations in $H^{2}\left(U^{2}\right)$ and generalizes an example I gave in [1].

EXAMPLE 1. Let $M_{n}$ denote the invariant subspace of $H^{2}\left(U^{2}\right)$ generated by the homogeneous polynomials of degree $n$. If $p\left(z_{1}, z_{2}\right)$ is a homogeneous polynomial of degree $n$ and $T_{p}$ is the operator of multiplication by $p$ and projection on $M_{n+1}^{\perp}$, then $\left\|T_{\nu}\right\|=\|p\|_{2}$, but the minimal norm of a dilation of $T_{p}$ which commutes with $S_{1}$ and $S_{2}$ is $\|p\|_{\infty}$.

The first statement comes from the fact that $T_{p}$ has rank 1. In fact, $T_{p} 1=p$ and $T_{p} x=0$ for $x \in M_{n+1}^{\perp} \ominus\{1\}$.

To prove the second statement, ${ }^{2}$ note that an operator $T$ on $H^{2}\left(U^{2}\right)$ which commutes with $S_{1}$ and $S_{2}$ and which is a dilation of $T_{p}$ must consist of multiplication by a function of the form $p+f$, where $f \in M_{n+1}$. Pick $\alpha=\left(\alpha_{1}, \alpha_{2}\right)$ with $\left|\alpha_{1}\right|=\left|\alpha_{2}\right|$ and $|p(\alpha)|=\|p\|_{\infty}$. If $h(\lambda)=p(\alpha)+f\left(\lambda \alpha_{1}, \lambda \alpha_{2}\right) / \lambda^{n}$, then $h$ is holomorphic in $|\lambda|<1$ and $|h(0)|=\|p\|_{\infty}$. We have

$$
\|T\|=\|p+f\|_{\infty} \geqq\|p(\lambda \alpha)+f(\lambda \alpha)\|_{\infty}
$$

where the last norm is the one variable $L^{\infty}$ norm. Further,

$$
\begin{aligned}
\|p(\lambda \alpha)+f(\lambda \alpha)\|_{\infty} & =\left\|\lambda^{n} p(\alpha)+f(\lambda \alpha)\right\|_{\infty}=\left\|\lambda^{n} h(\lambda)\right\|_{\infty} \\
& =\|h(\lambda)\|_{\infty} \geqq|h(0)|=\|p\|_{\infty} .
\end{aligned}
$$

This completes Example 1.

EXAMPLE 2. There is an invariant subspace $M$ of $H_{\mathscr{P}}^{2}\left(U^{2}\right)$ and an operator $T$ on $M$ commuting with $T_{1}$ and $T_{2}$ which has no bounded dilation $S$ which commutes with $S_{1}$ and $S_{2}$.

Let $x_{1}, x_{2}, \cdots$ be an orthonormal basis of $\mathscr{H}$, and let $M$ consist of functions of the form $\sum_{\Sigma} a_{n m} z_{1}^{n} z_{2}^{m}$ where $a_{n m}$ lies in the span of $x_{1}, x_{2}, \cdots$, $x_{n+m-1}$. Let $Q_{n}$ denote the projection of $\mathscr{H}$ on the span of $x_{n}$, and let $p_{1}, p_{2}, \cdots$ be homogeneous polynomials of degrees $1,2, \cdots$, which satisfy

$$
\sum\left\|p_{n}\right\|_{2}^{2}<\infty
$$

and

$$
\left\|p_{n}\right\|_{\infty} \rightarrow \infty
$$

For $x \in M^{\perp}$,

$$
x=\sum_{j, k=0}^{\Upsilon} a_{j k} z_{1}^{j} z_{2}^{k},
$$

\footnotetext{
2 This proof incorporates simplifications pointed out to me by $\mathrm{H}$. Alexander.
} 
where $a_{j k}$ is a linear combination of $x_{j+k}, x_{j+k+1}, \cdots$. Let $T_{n}^{\prime}$ denote the operator on $M^{\perp}$ of multiplication by $p_{n} Q_{n}$ and projection on $M^{\perp}$. Thus

$$
T_{n}^{\prime} x=P_{M \perp} \perp p_{n} \sum_{j+k \leqq n} z_{1}^{j} z_{2}^{k}\left(Q_{n} a_{j k}\right),
$$

and since $Q_{n} a_{j k}=\left\langle a_{j k}, x_{n}\right\rangle x_{n}$, we have $p_{n} z_{1}^{j} z_{2}^{k} Q_{n} a_{j k} \in M$ if $j+k>0$. It follows that

$$
T_{n}^{\prime} x=p_{n} Q_{n} a_{00}=\left\langle a_{00}, x_{n}\right\rangle p_{n} x_{n} .
$$

Clearly $T_{n}^{\prime} x \perp T_{m}^{\prime} x$ if $n \neq m$ and so, by (1) and (3), $T=\sum_{n=0}^{\infty} T_{n}^{\prime}$ exists in the strong operator topology and $T$ commutes with $T_{1}$ and $T_{2}$.

Now any (bounded) dilation $S$ of $T$ which commutes with $S_{1}$ and $S_{2}$ must have the form $S f=p\left(z_{1}, z_{2}\right) f$ where $p$ is an analytic function in $U^{2}$ whose values are operators on $\mathscr{H}$ and $\left\|p\left(z_{1}, z_{2}\right)\right\| \leqq K$, say. In addition, $S$ maps $M$ into $M$ and, if $f \in M^{\perp}, p\left(z_{1}, z_{2}\right) f=T f+x$, where $x \in M$. If $x \in M$, if $\langle\cdot, \cdot\rangle$ denotes the inner product in $\mathscr{H}$, and if $\left(z_{1}, z_{2}\right) \in U^{2}$, we have

It follows that

$$
\left\langle x\left(z_{1}, z_{2}\right), x_{n}\right\rangle=\sum_{j+k=n+1}^{\infty}\left\langle a_{j k}, x_{n}\right\rangle z_{1}^{j} z_{2}^{k} .
$$

$$
\begin{aligned}
K \geqq\left|\left\langle S x_{n}, x_{n}\right\rangle\right| & =\left|\left\langle T x_{n}, x_{n}\right\rangle+\left\langle x, x_{n}\right\rangle\right| \\
& =\left|p_{n}\left(z_{1}, z_{2}\right)+\sum_{j+k=n+1}^{\infty}\left\langle a_{j k}, x_{n}\right\rangle z_{1}^{j} z_{2}^{k}\right|
\end{aligned}
$$

for $\left(z_{1}, z_{2}\right) \in U$ and for all $n$. This contradicts Example 1 and (2).

EXAMPLE 3. There is an invariant subspace $M$ of $H^{2}\left(U^{3}\right)=H_{C}^{2}\left(U^{3}\right)$ and an operator $T$ on $M^{\perp}$ commuting with $T_{1}, T_{2}$ and $T_{3}$ which has no bounded dilation $S$ which commutes with $S_{1}, S_{2}$ and $S_{3}$.

Let $B(z)$ be a Blaschke product in one variable

$$
B(z)=\prod_{n=1}^{\infty} \frac{-\bar{a}_{n}}{\left|a_{n}\right|} \frac{z-a_{n}}{1-\bar{a}_{n} z},
$$

whose zeros are distinct but otherwise unspecified for the moment. Let

$$
B_{m}(z)=\prod_{n=m}^{\infty} \frac{-\bar{a}_{n}}{\left|a_{n}\right|} \frac{z-a_{n}}{1-\bar{a}_{n} z},
$$

and let $M_{n}$ be the invariant subspace of $H^{2}\left(U^{3}\right)$ generated by the homogeneous polynomials in $z_{1}$ and $z_{2}$ of degree $n$. Let $M$ denote the closure of the span of

$$
B_{1}\left(z_{3}\right) M_{1} \cup B_{2}\left(z_{3}\right) M_{2} \cup \cdots
$$


Thus $M$ is the invariant subspace of $H^{2}\left(U^{3}\right)$ generated by all functions of the form $B_{j}\left(z_{3}\right) p\left(z_{1}, z_{2}\right)$ where $p$ is a homogeneous polynomial of degree $j$.

Again we choose homogeneous polynomials $p_{0}, p_{1}, \cdots$ in $z_{1}, z_{2}$ of degrees $0,1, \cdots$ and satisfying (1) and (2). This time, $T_{n}^{\prime}$ is the operator on $M^{\perp}$ of multiplication by $p_{n-1} B_{n}$ and projection on $M^{\perp}$.

Clearly $T_{n}^{\prime} f=0$ (i.e. $p_{n-1} B_{n} f \in M$ ) if either $f \in M_{1}$ or $f\left(a_{n-1}\right)=0$. Thus $T_{n}^{\prime}$ has rank 1 and is zero on the orthogonal complement of the span of the function

Furthermore,

$$
F\left(z_{1}, z_{2}, z_{3}\right)=\left(1-\bar{a}_{n-1} z_{3}\right)^{-1}
$$

$$
T_{n}^{\prime} F=p_{n-1} B_{n}\left(1-\bar{a}_{n-1} z_{3}\right)^{-1}
$$

and

$$
\left\|p_{n-1} B_{n}\left(1-\bar{a}_{n-1} z_{3}\right)^{-1}\right\|=\left\|p_{n-1}\right\|\left\|\left(1-\bar{a}_{n-1} z_{3}\right)^{-1}\right\|,
$$

so that $\left\|T_{n}^{\prime}\right\| \leqq\left\|p_{n-1}\right\|$. In addition, (4) implies that the ranges of the $T_{n}^{\prime}$ are orthogonal, so we may conclude that $T=\sum_{n} T_{n}^{\prime}$ exists in the strong operator topology and commutes with $T_{1}, T_{2}$ and $T_{3}$. We claim there is no function $f \in M$ such that

$$
\left\|\sum_{n=1}^{\infty} p_{n-1}\left(z_{1}, z_{2}\right) B_{n}\left(z_{3}\right)+f\right\|_{\infty}=K<\infty .
$$

In fact, if $f \in M, f\left(z_{1}, z_{2}, a_{n}\right)$ has homogeneous degree at least $n+1$, so that, setting $z_{3}=a_{n}$ in (5) gives

$$
\left\|p_{n} B_{n+1}\left(a_{n}\right)+f\left(z_{1}, z_{2}, a_{n}\right)\right\|_{\infty} \leqq K
$$

and $f\left(z_{1}, z_{2}, a_{n}\right) \in M_{n+1}$. If we now assume that $B$ is chosen so that $\boldsymbol{B}_{n+1}\left(a_{n}\right)$ is bounded from 0 (i.e. if the sequence $\left\{a_{n}\right\}$ is interpolating) we have obtained a contradiction.

\section{REFERENCES}

1. D. N. Clark, On commuting contractions, J. Math. Anal. Appl. 32 (1970), 590-596. MR 42 \#2309.

2. S. Parrott, Unitary dilations for commuting contractions, Pacific J. Math. 34 (1970), 481-490. MR 42 \#3607.

3. D. E. Sarason, Generalized interpolation in $H^{\infty}$, Trans. Amer. Math. Soc. 127 (1967), 179-203. MR 34 \#8193.

4. B. Sz.-Nagy and C. Foiaş, Commutants de certains opérateurs, Acta. Sci. Math. (Szeged) 29 (1968), 1-17. MR 39 \#3346.

5. - Dilatation des commutants d'opérateurs, C. R. Acad. Sci. Paris Sér. A-B 266 (1968), A493-A495. MR 38 \#5049.

Department of Mathematics, University of California, los Angeles, CaliFORNIA 90024 